\title{
MULTIFREQUENCY SPECTRAL STUDIES OF SNRS
}

\section{X.Z. ZHANG}

Beijing Astronomical Observatory, CAS, Beijing 100080, P.R. China

X.J. WU

Beijing University, Beijing 100871, P.R. China

L.A. HIGGS AND T.L. LANDECKER

DRAO, P.O.Box 248, Penticton, B.C. V2A 6K3, Canada

D.A. GREEN

MRAO, Cambridge CB3 OHE, UK

AND

D.A. LEAHY

Department of Physics, The University of Calgary, T2N 1N4, Canada

\section{Introduction}

Radio supernova remnants(SNRs) of large angular diameter are obvious objects for multifrequency spectral studies from long wavelengths to short wavelengths, as resolutions at low frequency are usually about several arcminutes. An international cooperation $^{1}$ consists of MRAO, BAO, DRAO, MPI, and astronomers from Beijing University, Beijing Normal University, China and Calgary University, Canada. This international collaboration is also a part of the Panorramic Spectral Imaging of the Milky Way (PI R. Taylor ). Some results on HB21, G78.2+2.1, and HB9 are presented in this paper.

\section{Results}

\section{HB21}

The integral flux density of $\mathrm{HB} 21$ at $232 \mathrm{MHz}$ is about $390 \pm 30 \mathrm{Jy}$ measured from the new data. By combining this integral flux with that measured at $408 \mathrm{MHz}$ $(290 \pm 20 \mathrm{Jy})$ and $4750 \mathrm{MHz}(110 \pm 5 \mathrm{Jy})$ from the maps of Tatematsu(1990) and

${ }^{1}$ The project is supported by the National Natural Science Foundation of China. 
Reich(1983), integral spectral indices of $-0.41 \pm .02$ and $-0.39 \pm .02$ at frequency pairs of $232-4750 \mathrm{MHz}$ and $408-4750 \mathrm{MHz}$ are obtained respectively. A good linear fitting with the three flux densities together can be obtained.

T-T plot method was used to derive further the integral spectral index and spectral variations over the remnant. This method is independent of base level and is sensitive only to the variation of the temperature of object. The integral spectral index derived by this method is $-0.43 \pm .02$ between $232-4750 \mathrm{MHz}$ and $-0.44 \pm .02$ between $408-4750 \mathrm{MHz}$. All maps used here were convolved to resolution of $5.2^{\prime} \times 4.7^{\prime}$.

The mean spectral index is 2.55 at southeast, falling to 2.35 at northwest. A ring-shape structure of steeper spectra is found from the calculated results. It surrounds the central flat spectrum area which contains the $\mathrm{X}$-ray emission area. This is an interesting result to be discussed some where else.

\section{G78.2+2.1}

By using the same T-T plot method, the integral spectral indices of G78.2+2.1 and the mean indices in 12 boxes within the remnant were calculated respectively. From these results, two conclusions can be derived. The first is that spectral indices in each box in frequency range $232-2695 \mathrm{MHz}$ are almost same as that in same box in frequency range $408-4750 \mathrm{MHz}$. The maximum diffusion of the spectral indices to their mean value is 0.12 in the box 9 , while the mean-diffusion is 0.05 .

The numbers suggest that the indices measured by this method are true, because of the small diffusion. The second conclusion is that, for the most case, spectral indices of frequency pairs $232-1420 \mathrm{MHz}$ and $1420-4750 \mathrm{MHz}$ in each box show flat-steep-relationships. Also the variations of spectral indices in low frequency range, $232-1420 \mathrm{MHz}$, show some distribution which is more far from the galactic plane, more flat spectra are found, whereas the spectral indices in frequencty range $1420-4750 \mathrm{MHz}$ do not show the tendency. Same phenomenon is also found in the field of HB21. The reason may be same. That is more far from the galactic plane more less the contribution of the galactic background emission which has property of steep spectrum.

\section{HB9}

Spectral index distribution of HB9 were calculated in three frequency pairs, i.e. $151-1420 \mathrm{MHz}, 232-2695 \mathrm{MHz}$, and $408-4750 \mathrm{MHz}$. The maps used here has been convolved to $8^{\prime} \times 8^{\prime}$ beam size and maps were divided into 16 boxes which are same to that in the paper of Leahy D.A. and Roger R.(1991). Spectrum of HB9 in the boxes $\mathrm{J}, \mathrm{K}, \mathrm{P}$ have different property from other region. In the east and south of the remnant spectrum is steeper than that in west and north part. The diffusion of spectral indices of the three frequency pairs are also large in west and north region of the remnant.

\section{References}

Leahy, D.A. et al., 1991, AJ, 101, 1033

Reich, W., Furst, E. and Sieber, 1983, IAU Symposium 101, 377

Tatematsu, K., Fukui, Y., Landecker, T.L., and Roger, R.S., 1990, A\&AP,1990, 237, 189

Wendker, H.J. et al., 1991, A\&A, 241, 551 